03

\title{
Квазиоптическое уравнение в средах со слабым поглощением
}

\author{
(c) Н.Н. Розанов ${ }^{1,2,3}$ \\ ${ }^{1}$ Государственный оптический институт им. С.И. Вавилова, \\ 199053 Санкт-Петербург, Россия \\ 2 Физико-технический институт им. А.Ф. Иоффре, \\ 194021 Санкт-Петербург, Россия \\ ${ }^{3}$ Университет ИТМО, \\ 197101 Санкт-Петербург, Россия \\ e-mail: nnrosanov@mail.ru
}

Поступила в редакцию 25.03.2019 г.

В окончательной редакции 25.03.2019 г.

Принята к публикации 16.04.2019 г.

\begin{abstract}
Проведен анализ вида квазиоптического уравнения для пучка монохроматического излучения, распространяющегося через слой слабо поглощающей линейной среды. Сделан вывод о необходимости модификации стандартного вида этого уравнения с использованием „эффективного коэффициента диффузии“.
\end{abstract}

Ключевые слова: Квазиоптическое приближение, метод медленно меняющейся огибающей, эффективный коэффициент диффузии.

DOI: $10.21883 /$ OS.2019.08.48042.122-19

Квазиоптическое приближение и соответствующее квазиоптическое (другие названия - параксиальное и параболическое) уравнение, справедливое для пучков и импульсов электромагнитного излучения, близких к однонаправленной монохроматической плоской волне [1$3]$, широко используются как в линейной, так и нелинейной оптике [4-6]. Это приближение особенно эффективно для описания распространения лазерного излучения, обладающего узким частотным спектром (ширина линии много меньше несущей частоты) и малой (близкой к дифракционной) угловой расходимостью.

Естественно, что оптическое излучение распространяется на достаточно большие длины в средах, если поглощение в них мало. Ввиду этого в большинстве применений квазиоптического уравнения поглощением в среде пренебрегают. Тем не менее даже слабые диссипативные факторы - усиление или поглощение - могут приводить к ряду важных эффектов в линейных $[7,8]$ и нелинейных [6] средах.

В настоящей работе мы иллюстрируем этот тезис на простейшем примере распространения пучка монохроматического излучения через слой однородной линейной слабо поглощающей среды. Поглощение приводит к тому, что на выходе из слоя составляющие пучок наклонные парциальные плоские волны, длина пробега которых в слое больше, чем для осевой волны, ослабевают соответственно сильнее, чем осевые. Тем самым геометрический фактор вносит в схему определенную анизотропию поглощения - его зависимость от направления распространения волны (обобщенный дихроизм). Заметим, что для истинно анизотропных сред с дихроизмом [9] само объемное поглощение меняется вследствие изменения поляризации излучения с наклоном фронта волны, но это изменение зависит от выбора поперечного направления наклона.

Будем исходить из скалярного уравнения Гельмгольца (излучение близко к линейно поляризованному) для напряженности электрического поля излучения $\widetilde{E}$

$$
\Delta \widetilde{E}+k^{2} \widetilde{E}=0 .
$$

Здесь $\Delta=\frac{\partial^{2}}{\partial x^{2}}+\frac{\partial^{2}}{\partial y^{2}}+\frac{\partial^{2}}{\partial z^{2}}-$ оператор Лапласа и $k=\frac{\omega}{c} n-$ комплексное волновое число, выражающееся через комплексный показатель преломления среды $n=n^{\prime}+i n^{\prime \prime}, \omega-$ частота излучения, $c-$ скорость света в вакууме, $k^{\prime}=\operatorname{Re} k=\frac{\omega}{c} n^{\prime}, k^{\prime \prime}=\operatorname{Im} k=\frac{\omega}{c} n^{\prime \prime}, x$, у и $z$ - декартовы координаты. В рассматриваемом случае $n^{\prime}=\operatorname{Re} n>0, \quad n^{\prime \prime}=\operatorname{Im} n>0$ (поглощение) и $n^{\prime \prime} \ll n^{\prime}$ (поглощение слабо). Пусть пучок излучения распространяется преимущественно вдоль нормали к слою $z$. Грани слоя считаем просветленными (отражение отсутствует). В квазиоптическом методе медленно меняющаяся огибающая $E$ обычно вводится подстановкой (временной множитель, отвечающий гармоническому изменению поля по времени с частотой $\omega$, опущен)

$$
\widetilde{E}=\operatorname{Re}\left[E \exp \left(i k^{\prime} z\right)\right] .
$$

Тогда с учетом медленности изменения $E$ (по сравнению с фигурирующей в (2) экспонентой) и пренебрегая квадратичным по коэффициенту поглощения членом $k^{\prime \prime 2}$, получим из (1)

$$
2 i k^{\prime} \frac{\partial E}{\partial z}+\Delta_{\perp} E+2 i k^{\prime} k^{\prime \prime} E=0
$$

где $\Delta_{\perp}=\frac{\partial^{2}}{\partial x^{2}}+\frac{\partial^{2}}{\partial y^{2}}-$ поперечный оператор Лапласа. Для прозрачных сред $k^{\prime \prime}=0$, так что последний член в левой части (3) отсутствует. 
Пучок излучения можно разложить в спектр парциальных плоских волн с вещественными поперечными волновыми векторами $\mathbf{k}_{\perp}=\left(k_{x}, k_{y}\right), k_{\perp}^{2} \ll k^{\prime 2}$, что отвечает малости угла наклона их распространения по отношению к оси слоя $\theta_{x, y} \approx k_{x, y} / k^{\prime}$. Для таких волн из (3) следует

$$
2 i k^{\prime} \frac{d E}{d z}=\left(k_{\perp}^{2}-2 i k^{\prime} k^{\prime \prime}\right) E .
$$

Согласно (4), вещественная амплитуда волны (квадратный корень из ее интенсивности) $A=|E|$ убывает с ростом $z$ независимо от направления распространения волны:

$$
A(z)=A(0) \exp \left(-k^{\prime \prime} z\right)
$$

(от наклона к оси слоя зависит только фазовый набег волны). Тем самым в этом приближении теряется отмеченный выше один из основных эффектов поглощения.

Положение дел меняется при переопределении медленно меняющейся огибающей $E$, если вместо (2) использовать подстановку

$$
\widetilde{E}=\operatorname{Re}[E \exp (i k z)]
$$

Тогда из (1) получим

$$
2 i\left(k^{\prime}+i k^{\prime \prime}\right) \frac{\partial E}{\partial z}+\Delta_{\perp} E=0
$$

или

$$
2 i k^{\prime} \frac{\partial E}{\partial z}+(1-i d) \Delta_{\perp} E=0 .
$$

Здесь $d=\frac{n^{\prime \prime}}{n^{\prime}}-$ „эффективный коэффициент диффузии“, вызванной поглощением и дополняющей обычную дифракцию в прозрачных средах $(0<d \ll 1)$.

Из (8) для парциальных волн с поперечным волновым вектором $\mathbf{k}_{\perp}$ с учетом (6) для вещественной амплитуды поля следует

$$
\begin{aligned}
A(z) & =|E(z)| \exp \left(-k^{\prime \prime} z\right) \\
& =A(0) \exp \left(-d \frac{k_{\perp}^{2}}{2 k^{\prime}} z\right) \exp \left(-k^{\prime \prime} z\right) .
\end{aligned}
$$

Видно, что по сравнению с (5) в (9) появляется дополнительный множитель, зависящий от направления распространения волны, и эта зависимость с учетом соотношения $k_{\perp}^{2} \ll k^{\prime 2}$ согласуется с точным решением применительно к ослаблению наклонно распространяющихся волн. Заметим также, что для локализованных пучков (с конечной мощностью) из (7) независимо от наличия или отсутствия поглощения следует сохранение линейной по амплитуде поля интегральной величины

$$
S_{2}=\iint E d x d y, \quad \frac{d S_{2}}{d z}=0 .
$$

Общее решение (7) или (8) имеет тот же вид, что и для прозрачной среды [4] с заменой вещественного волнового числа на комплексное. Это же относится и к виду гауссовых пучков и их обобщений. Такой подход применим, с одной стороны, для сред со слабым поглощением (в противном случае говорить о распространении излучения не приходится). С другой стороны, поправки за счет поглощения должны превосходить поправки более высокого порядка квазиоптического приближения, что для пучка с характерной шириной $w$ ведет к требованию $\left|n^{\prime \prime} / n^{\prime}\right|>\left(k^{\prime} w\right)^{-2}$.

Для сред с усилением $n^{\prime \prime}<0$, и для них (9) описывает большее усиление наклонных волн, проходящих в слое усиливающей среды большее расстояние, чем волны, распространяющиеся вдоль оси слоя. В практически важных случаях соответствующий рост угловой расходимости излучения неблагоприятен и имеются факторы, ограничивающие этот рост, включая угловую фильтрацию, апертурные ограничения и нелинейность усиления. Оставаясь в рамках линейной оптики, рассмотрим систему, состоящую из двух слоев (помечаемых далее индексами 1 и 2), обладающих толщинами $L_{1}$ и $L_{2}$ и комплексными показателями преломления $n_{1}$ и $n_{2}$; границы слоев вновь считаем просветленными. Тогда при условии $L_{1} n_{1}^{\prime \prime}+L_{2} n_{2}^{\prime \prime}=0$ волны с осевым направлением распространения не ослабляются после прохождения двух слоев. Одновременно для распространяющихся наклонно волн можно добиться ослабления, возрастающего с ростом наклона. Такая пара слоев может быть заменена одним с эффективными характеристиками $n^{\prime \prime}=0$ и $d>0$. Соответственно для описания этой и подобных систем (с бо́льшим числом слоев) целесообразно использовать обобщение квазиоптического уравнения типа (8). Наличие в (8) „эффективного коэффициента диффузии“ имеет принципиальное значение для устойчивости вихревых солитонов в лазерах с насыщающимся поглощением [6] и существенно проявляется в других задачах нелинейной оптики, описываемых квазиоптическим уравнением с введением в него соответствующих дополнительных нелинейных членов.

\section{Конфликт интересов}

Автор заявляет, что у него нет конфликта интересов.

\section{Список литературы}

[1] Леонтович М.А. // Изв. АН СССР, сер. физ. 1944. Т. 8. C. $16-22$.

[2] Леонтович М.А., Фок В.А. // ЖЭТФ. 1946. Т. 16. № 7. C. $557-573$.

[3] Фок В.А. Проблемы диффракции и распространения электромагнитных волн. М.: Сов. радио, 1970.

[4] Виноградова М.Б., Руденко О.В., Сухоруков А.П. Теория волн. М.: Наука, 1979.

[5] Власов С.Н., Таланов В.И. Самофокусировка волн. Н. Новгород: ИПФ РАН, 1997.

[6] Розанов Н.Н. Диссипативные оптические солитоны. М.: Физматлит, 2011. 
[7] Розанов Н.Н. // Опт. и спектр. 2001. Т. 90. № 1. C. 136-138; Rozanov N.N. // Opt. Spectrosc. 2001. V. 90. N 1. P. $121-123$.

[8] Розанов Н.Н. // Опт. и спектр. 2001. Т. 90. № 2. C. 311-314; Rozanov N.N. // Opt. Spectrosc. 2001. V. 90. N 2. P. 26-268.

[9] Борн М., Воль $\oint$ Э. Основы оптики. М.: Наука, 1973; перевод с англ. М. Born, E. Wolf. Principles of Optics. Oxford: Pergamon, 1969. 